\title{
ARTICLE OPEN Obesity and survival among a cohort of breast cancer patients is partially mediated by tumor characteristics
}

Cindy K. Blair ${ }^{1,2}$, Charles L. Wiggins (D) ${ }^{1,2}$, Andrea M. Nibbe ${ }^{1,2}$, Curt B. Storlie ${ }^{3}$, Eric R. Prossnitz (D) ${ }^{1,2}$, Melanie Royce (iD) Lesley C. Lomo iD ${ }^{4}$ and Deirdre A. Hill ${ }^{1,2 *}$

Obesity exerts adverse effects on breast cancer survival, but the means have not been fully elucidated. We evaluated obesity as a contributor to breast cancer survival according to tumor molecular subtypes in a population-based case-cohort study using data from the Surveillance Epidemiology and End Results (SEER) program. We determined whether obese women were more likely to be diagnosed with poor prognosis tumor characteristics and quantified the contribution of obesity to survival. Hazard ratios (HRs) and 95\% confidence intervals (Cl) were calculated via Cox multivariate models. The effect of obesity on survival was evaluated among 859 incident breast cancers (subcohort; 15\% random sample; median survival 7.8 years) and 697 deaths from breast cancer (cases; $100 \%$ sample). Obese women had a 1.7- and 1.8-fold increased risk of stage III/IV disease and grade 3/4 tumors, respectively. Obese women with Luminal A- and Luminal B-like breast cancer were $1.8(95 \% \mathrm{Cl} 1.3-2.5)$ and $2.2(95 \% \mathrm{Cl} 0.9-5.0)$ times more likely to die from their cancer compared to normal weight women. In mediation analyses, the proportion of excess mortality attributable to tumor characteristics was $36.1 \%$ overall and $41 \%$ and $38 \%$ for Luminal A- and Luminal B-like disease, respectively. Obesity was not associated with breast cancer-specific mortality among women who had Her2-overexpressing or triple-negative tumors. Obesity may influence hormone-positive breast cancer-specific mortality in part through fostering poor prognosis tumors. When tumor biology is considered as part of the causal pathway, the public health impact of obesity on breast cancer survival may be greater than previously estimated.

npj Breast Cancer (2019)5:33

; https://doi.org/10.1038/s41523-019-0128-4

\section{INTRODUCTION}

Currently in the U.S., almost $40 \%$ of the adult population is obese, and another $30 \%$ is overweight. ${ }^{1,2}$ Obesity is one of the most prevalent modifiable risk factors for chronic disease and has received considerable attention in relation to cancer outcomes. However, despite numerous studies that have evaluated obesity and breast cancer-specific mortality, the means through which obesity exerts its effects on breast cancer survival have not been fully elucidated.

In recent meta-analyses, breast cancer-specific death among obese women is elevated 1.3-fold compared to normal weight women. ${ }^{3,4}$ However, in most studies, risk estimates for the relationship between body mass index (BMI) and breast cancer mortality have been adjusted for tumor characteristics (stage at diagnosis and/or tumor size, grade, and nodal status). Evidence is accumulating that tumor characteristics may be on the causal pathway (potential mediators) between BMI and cancer mortality. ${ }^{5-7}$ If so, then the BMI-mortality risk estimates adjusted for these factors is likely to be underestimated. Statistical adjustment for a causal intermediate can substantially attenuate risk estimates or even result in a reversal of direction (suggested reduced risk). ${ }^{8}$

In a large population-based case-cohort study, we investigated the role of obesity and associated mechanisms on breast cancerspecific death. We first examined whether higher BMI was associated with more aggressive tumor characteristics. We then investigated the relationship between higher BMI and breast cancer survival, with consideration of the possibility that tumor characteristics were on the causal pathway, often termed "mediators." We evaluated the BMI-mortality relationship according to breast cancer subtypes, as results from previous studies have suggested that the effect of BMI may be stronger in luminal disease. ${ }^{9-14}$

\section{RESULTS}

Population characteristics

The analytic case-cohort consisted of 697 deaths from breast cancer (cases) and 859 breast cancers (subcohort; 15\% random sample weighted by $6.67 \times(1 /$ sampling fraction) in the analysis) (Fig. 1). Median follow-up in the subcohort was 94 months (range 2-205 months). Women who died from breast cancer were more likely to be Hispanic/Latina ( $33 \%$ vs. $23 \%$ ), obese (33\% vs. $24 \%$ ), and to be diagnosed with later stage tumors (stage III/IV; $50 \%$ vs. $17 \%$ ) as well as more aggressive tumor subtypes (non-Luminal $A$ like; $40 \%$ vs. $26 \%$ ), compared to the subcohort (Table 1 -before imputation; Supplementary Table 1-after imputation).

\section{Mediation analysis}

Tumor characteristics differed by BMI (Table 2). Women who were obese were more likely to be diagnosed with stage 3/4 disease, and particularly, metastases, as well as grade $3 / 4$ tumors, relative to normal weight women. Obese women were also 2-fold more likely to have $\geq 2$ comorbidities and 1.5 -fold more likely to receive chemotherapy than normal weight women.

Overweight women did not have an elevated risk of breast cancer-specific mortality. In contrast, obese women had 1.63-fold overall increased risk (Table 3-HR1), compared to normal weight

\footnotetext{
${ }^{1}$ Department of Internal Medicine, University of New Mexico, Albuquerque, NM, USA. ${ }^{2}$ University of New Mexico Comprehensive Cancer Center, Albuquerque, NM, USA. ${ }^{3}$ Department of Biomedical Statistics and Informatics, Mayo Clinic College of Medicine, Rochester, MN, USA. ${ }^{4}$ Department of Pathology, University of Utah, Salt Lake City, UT, USA. *email: DAHill@salud.unm.edu
} 
Cases (all breast cancer specific deaths) $n=1,049$

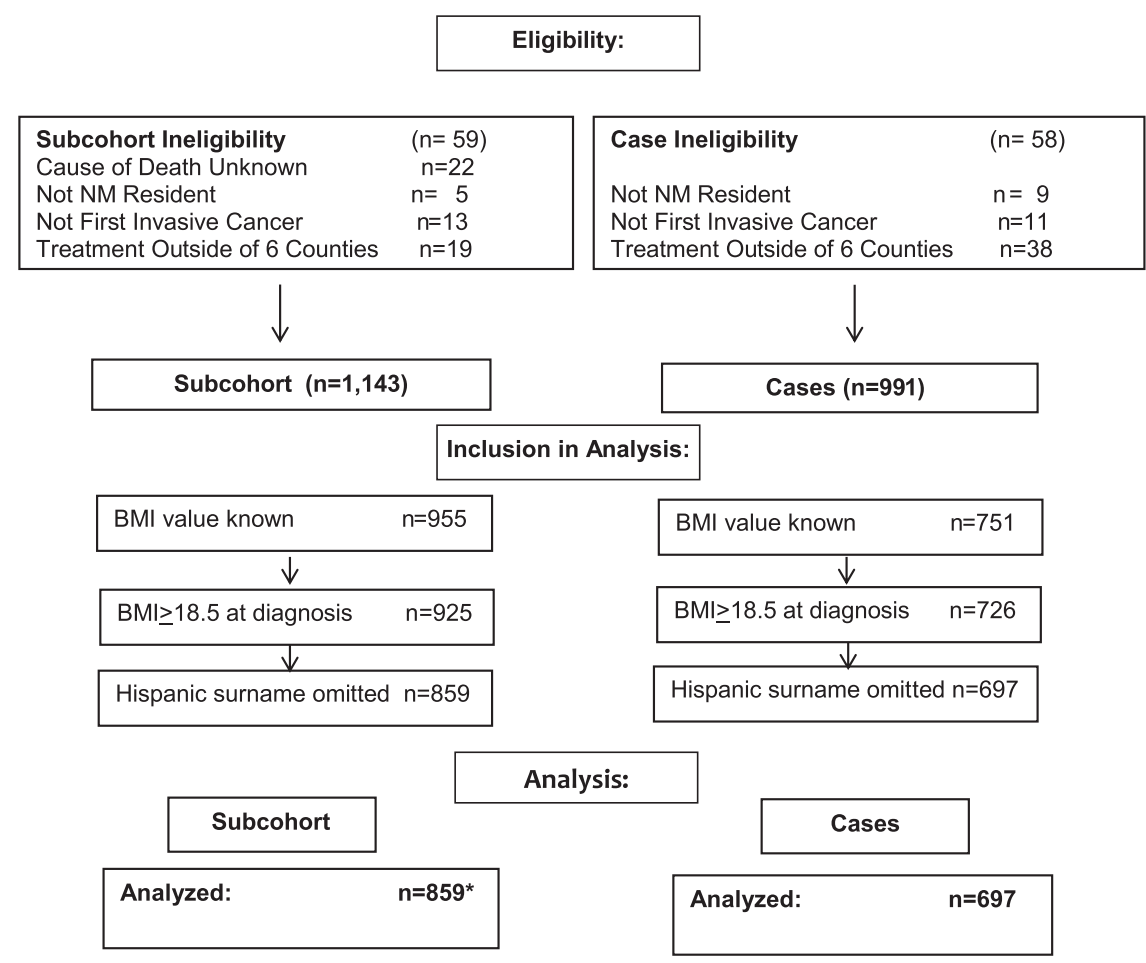

${ }^{*} \mathrm{~N}=113(13.2 \%)$ of women selected from the cohort are also cases and are included in

the case total of $n=697$, according to standard statistical case-cohort study methodology.

Fig. 1 Study flow diagram of a population-based case-cohort study, including cases (all deaths due to breast cancer-related causes) and a subcohort (a 15\% random sample of all other eligible breast cancers) that was used to examine the relationship between body mass index and breast cancer-specific mortality

women. Obese women also had a 1.78-2.16-fold increased breast cancer mortality risk within subgroups of Luminal A- and Luminal B-like disease, respectively (Table 3-HR1; Fig. 2). However, there was little evidence for an association between obesity and mortality among women who had Her2-overexpressing or triplenegative tumors. Premenopausal obese women had statistically non-significantly elevated hazard ratios (HRs) for breast cancer mortality in luminal tumors than postmenopausal obese women, but mortality was not elevated for non-luminal tumors in either menopausal status group (Supplementary Table 2). The effects of obesity and luminal vs. non-luminal subtype on breast cancer survival did not exceed that expected from their joint multiplicative effects ( $p$ value for interaction $=0.08$ ).

Statistical adjustment for tumor characteristics substantially reduced the relationship between $\mathrm{BMI}$ and mortality for luminal tumors (Table 3-HR2). This suggests that these factors have a limited influence on the BMI-mortality relationship after accounting for tumor stage and grade. The proportion of the obesity-mortality relationship mediated by poor prognosis tumor characteristics was $41.4 \%$ for Luminal A-like and $37.7 \%$ for Luminal B-like subtypes (see p. 493 in ref. ${ }^{15}$ ). Thus the age- and ethnicityadjusted model (Table 3-HR1) provides an unbiased estimate of the BMI effect on breast cancer mortality, unadjusted for factors that appear to mediate such effects.

\section{DISCUSSION}

Our large, population-based case-cohort study afforded the opportunity to investigate the means whereby BMI exerts influence on breast cancer-specific mortality. Obesity appears to influence breast cancer survival in part by fostering greater tumor size, higher grade, and a substantially increased risk of metastases. We found that $36 \%$ of the increased mortality risk overall due to obesity was potentially attributable to (or mediated by) tumor characteristics at diagnosis and $38-41 \%$ for luminal subtypes. Thus the proportion of the effect of obesity on breast cancer survival that acts through (is mediated by) tumor biology is high.

To date, the increased mortality risk in obese women has been identified predominantly in ER+/luminal subtypes, ${ }^{9-14}$ consistent with our findings. Relative risk estimates in those studies have ranged from 1.2-1.5, lower than the 1.7-1.8-fold we report. This difference, albeit modest, is possibly in part due to attenuation via adjustment for tumor characteristics (HR2, Table 3) or other potential mediators. Our results contrast somewhat with studies in which obesity-associated mortality risk was elevated in women with triple-negative tumors. ${ }^{16-19}$ Of note, those studies included all-cause mortality as a component or sole endpoint. BMI is expected to be related to all-cause mortality in part due to cardiovascular and other chronic disease mortality even if minimal or no relationship with breast cancer-specific survival.

Our results should be interpreted in light of study limitations and strengths. The smaller sample size constrained our ability to provide precise effect estimates for non-luminal subtypes. Weight loss at diagnosis, especially if non-intentional, could have influenced our results. Strengths of our population-based study include a case-cohort design with nearly 700 deaths from breast cancer, facilitating risk estimates for Luminal A and Luminal B breast cancer subtypes. 
Table 1. Characteristics of included women diagnosed with incident invasive breast cancer in six New Mexico counties

\begin{tabular}{|c|c|c|c|c|}
\hline \multirow[t]{2}{*}{ Characteristic } & \multicolumn{2}{|c|}{$\begin{array}{l}\text { Subcohort } \\
(N=859) 15 \% \\
\text { random sample }\end{array}$} & \multicolumn{2}{|c|}{$\begin{array}{l}\text { Breast cancer } \\
\text { deaths } \\
(N=697) \\
100 \% \text { sample }\end{array}$} \\
\hline & $\mathrm{N}$ & (\%) & $\mathrm{N}$ & $(\%)$ \\
\hline \multicolumn{5}{|l|}{ Age at diagnosis (years) } \\
\hline$<40$ & 43 & 5.0 & 56 & 8.1 \\
\hline $40-49$ & 146 & 17.0 & 143 & 20.5 \\
\hline $50-59$ & 217 & 25.2 & 180 & 25.8 \\
\hline $60-69$ & 206 & 24.0 & 117 & 16.8 \\
\hline $70-79$ & 158 & 18.4 & 123 & 17.6 \\
\hline $80+$ & 89 & 10.4 & 78 & 11.2 \\
\hline \multicolumn{5}{|l|}{ Year of diagnosis } \\
\hline 1997-2000 & 253 & 29.4 & 257 & 36.9 \\
\hline 2001-2004 & 256 & 29.8 & 238 & 34.1 \\
\hline 2005-2009 & 350 & 40.8 & 202 & 30.0 \\
\hline \multicolumn{5}{|l|}{ Race/ethnicity } \\
\hline Non-Hispanic white & 661 & 76.9 & 464 & 66.6 \\
\hline Hispanic/Latina & 198 & 23.1 & 233 & 33.4 \\
\hline \multicolumn{5}{|l|}{ Menopausal status $^{a}$} \\
\hline Pre/peri & 160 & 18.6 & 168 & 24.1 \\
\hline Postmenopausal & 666 & 77.5 & 497 & 71.3 \\
\hline Missing & 33 & 6.2 & 32 & 4.6 \\
\hline \multicolumn{5}{|c|}{ Body mass index $\left(\mathrm{kg} / \mathrm{m}^{2}\right)^{\mathrm{a}}$} \\
\hline $18.5-24.9$ & 338 & 39.3 & 252 & 36.2 \\
\hline $25.0-29.9$ & 246 & 28.6 & 172 & 24.7 \\
\hline$>30.0$ & 195 & 22.7 & 211 & 30.3 \\
\hline Missing & 80 & 9.3 & 62 & 8.9 \\
\hline \multicolumn{5}{|l|}{ Stage $^{a}$} \\
\hline 1 & 382 & 44.5 & 94 & 13.5 \\
\hline 2 & 281 & 32.7 & 222 & 31.9 \\
\hline $3 / 4$ & 133 & 15.5 & 307 & 44.0 \\
\hline Missing & 63 & 7.3 & 74 & 10.6 \\
\hline \multicolumn{5}{|l|}{ Tumor subtype ${ }^{a}$} \\
\hline Luminal A & 526 & 61.2 & 320 & 45.9 \\
\hline Luminal B & 91 & 10.6 & 105 & 15.1 \\
\hline Her2+ ER-/PR- & 24 & 2.8 & 32 & 4.6 \\
\hline Triple negative & 61 & 7.1 & 85 & 12.2 \\
\hline Missing & 157 & 18.3 & 155 & 22.2 \\
\hline \multicolumn{5}{|l|}{ Treatment (yes) ${ }^{\mathrm{b}}$} \\
\hline Chemotherapy & 381 & 44.4 & 469 & 67.3 \\
\hline Radiation & 560 & 65.2 & 400 & 57.4 \\
\hline Endocrine therapy & 586 & 68.2 & 418 & 60.0 \\
\hline \multicolumn{5}{|c|}{$\begin{array}{l}E R \text { estrogen receptor, Her2 human epidermal growth factor receptor } 2, \\
P R \text { progesterone receptor, Pre/peri premenopausal/perimenopausal } \\
{ }^{\text {a }} \text { The table reflects the frequencies prior to imputation, thus missing data } \\
\text { are shown, which reflect the number of imputed values for each variable } \\
\text { b Non-exclusive categories }\end{array}$} \\
\hline
\end{tabular}

The findings position obesity as a potential driver of poor prognosis tumor biology. Our results imply that excess body weight may contribute to higher tumor stage and grade, including a greater incidence of metastases, specifically in the $\sim 80 \%$ of breast tumors that are considered luminal. Obese women have had aggressive tumor characteristics in previous investigations ${ }^{13,19-23}$; however, such factors have been considered confounders and not identified as intermediates on the causal pathway and thus strong mediators of disease outcomes. Such an analysis will attenuate measures of clinical and public health impact, which is of concern because obesity may be one of few modifiable risk factors for breast cancer prognosis. Whether weight loss after a diagnosis has beneficial effects on cancer outcomes is unknown and may depend in part on whether the direct and indirect effects of obesity exposure are reversible. Currently, several randomized controlled trials are evaluating the effects of weight loss among breast cancer survivors on cancer recurrence and long-term outcomes. ${ }^{24,25}$

Previous and current evidence linking obesity and poor prognosis tumor characteristics provide a foundation for understanding tumor characteristics as mediators of the BMI-breast cancer survival relationship. Biological mechanisms linking excess adiposity to poor prognosis tumor characteristics include local and systemic alterations to inflammatory markers, steroid hormones, cytokines/adipokines, insulin, and insulin-like growth factors (IGFs). The local and systemic alterations may directly affect cancer cells via activation of signaling pathways (e.g., estrogen, insulin/IGF, Janus-activated kinase/signal transducer and activator of transcription factor, etc.) or alterations in cellular metabolism or can indirectly affect the tumor microenvironment to promote cell proliferation, angiogenesis, and invasion. ${ }^{26-28}$ In addition, obese women have had greater tumor proliferation in several studies. $^{21,29,30}$ Such data do not establish that BMI contributes to adverse tumor characteristics directly. Alternative explanations for poor prognosis tumor characteristics include the possibility that initial cancer detection is delayed in obese women ${ }^{31-33}$ and that delayed detection of recurrence and metastases further contribute to survival, but that does not account for the restriction of many findings, including ours, to luminal tumors.

The lack of association between obesity and survival of nonluminal cancers is puzzling, as such tumors are more likely to have a poor prognosis. Despite being one of the larger populationbased studies to examine obesity and breast cancer-specific mortality, assessment of some relationships with non-luminal tumors were limited by cell sizes in this study. However, local and systemic tissue-specific mechanisms that link obesity with progression and survival may plausibly support greater growth and proliferation in hormone receptor-positive breast cancers. The occurrence and severity of breast adipose tissue inflammation is higher in obese compared to normal weight women ${ }^{34}$ and in postmenopausal relative to premenopausal women. ${ }^{35,36}$ Breast adipose tissue inflammation is associated with elevated aromatase levels, the rate-limiting enzyme in estrogen biosynthesis, ${ }^{37}$ and similar inflammation has been noted at distant sites. ${ }^{38,39}$ Thus increased estrogen production arising from aromatase activity, particularly in the postmenopausal environment, may selectively drive proliferation in estrogen-dependent breast cancer. Biosynthesis of estrogen may also reduce the effectiveness of endocrine therapy among obese women with hormone-positive subtypes. ${ }^{37}$ These cellular processes lend support to the dichotomy between luminal and non-luminal disease evident in this study. Less is known regarding other possible mechanisms linking obesity to progression and survival of non-hormone-related subtypes; however, this is an active area of research.

Whether poorer prognosis tumor characteristics are an effect of adiposity, delayed diagnosis, metabolic changes, or other or combined mechanisms, the clinical effect of obesity will be attenuated by statistical adjustment for factors that are a consequence of exposure. Thus the effect of BMI on breast cancer outcomes is likely to be somewhat stronger than previously reported. Our findings that BMI may influence tumor characteristics have implications for tumors beyond breast cancer. 
Table 2. Overweight and obesity in relation to tumor characteristics, Charlson comorbidity index, and treatment: cross-sectional analysis in a population-based subcohort $(n=859)$

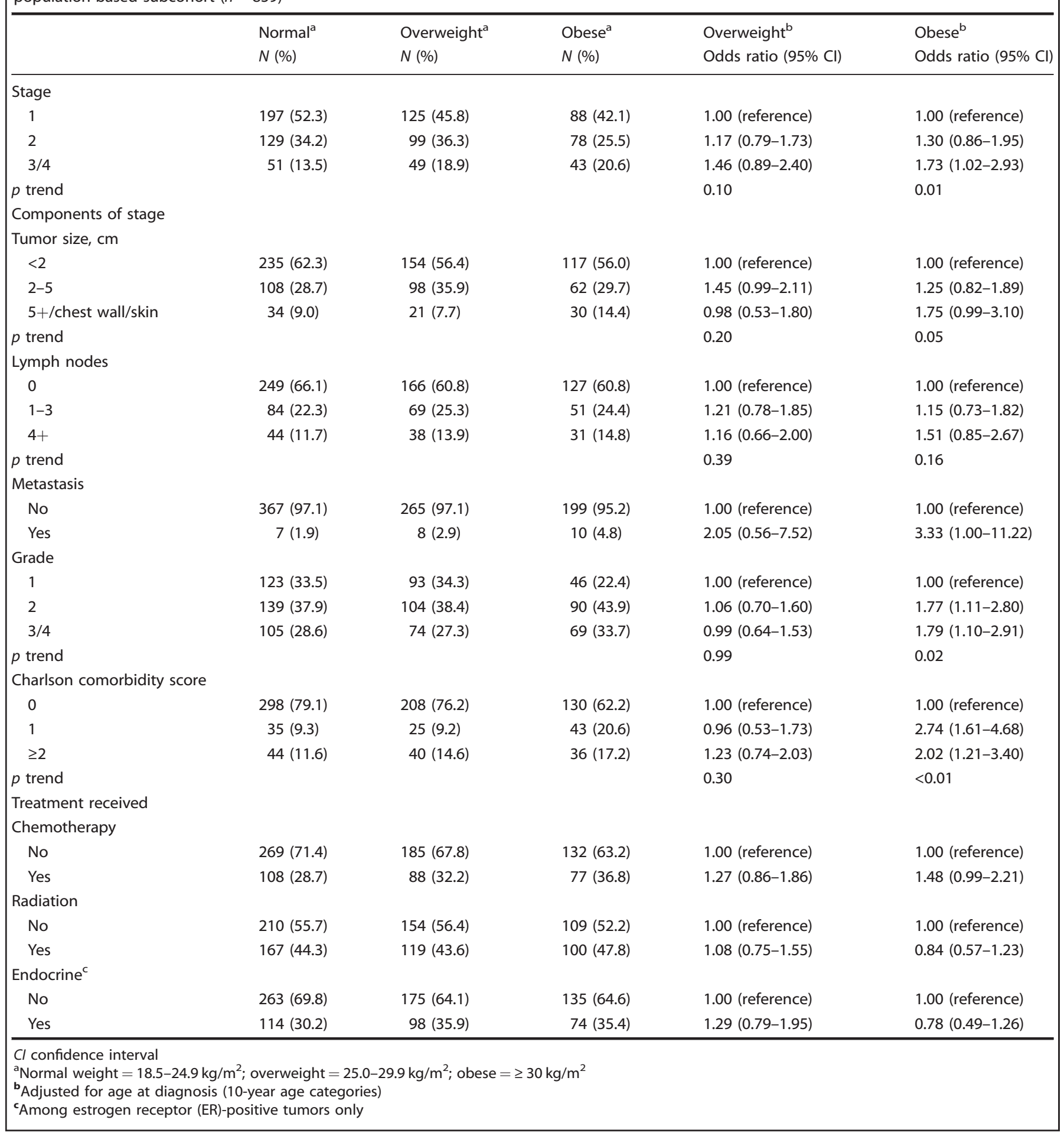

\section{METHODS}

Study design and population

Our population-based case-cohort study of contributors to breast cancer survival has been previously described. ${ }^{40,41}$ Eligible were Hispanic white and non-Hispanic white women diagnosed with invasive breast cancer between 1997 and 2009 in six New Mexico counties (representing 50\% of the New Mexico population). Breast cancer cases were identified through the New Mexico Tumor Registry, a founding member of the Surveillance, Epidemiology, and End Results program. To capture complete treatment information, only women who received treatment within the six county geographic area were eligible. Hispanic ethnicity was classified according to the North American Association of Central Cancer Registries algorithm. Cases identified solely by autopsy or death certificate and women with Hispanic ethnicity defined only by surname were excluded. The study population included all deaths attributed to breast cancer as an underlying cause on the death certificate, and a $15 \%$ random sample of all eligible breast cancers. Analytic models were restricted to women with $\mathrm{BMI} \geq$ $18.5 \mathrm{~kg} / \mathrm{m}^{2}$ (Fig. 1). 


\begin{tabular}{|c|c|c|c|c|c|}
\hline BMI category ${ }^{a}$ & Cohort, $N=859 ; N(\%)$ & Deaths, $N=697 ; N(\%)$ & $\mathrm{HR} 1(95 \% \mathrm{Cl})^{\mathrm{b}}$ & $\mathrm{HR} 2(95 \% \mathrm{Cl})^{\mathrm{C}}$ & Mediation proportion \\
\hline \multicolumn{6}{|l|}{ Overall } \\
\hline Overweight & $273(31.8)$ & $185(26.5)$ & $0.98(0.76-1.27)$ & $0.86(0.61-1.20)$ & \\
\hline Obese & $209(24.3)$ & $233(33.4)$ & $1.63(1.26-2.11)$ & $1.33(0.95-1.84)$ & $36.1 \%$ \\
\hline Overweight & $203(31.8)$ & $102(25.0)$ & $0.94(0.67-1.32)$ & $0.76(0.50-1.16)$ & \\
\hline Obese & $150(23.4)$ & $140(34.3)$ & $1.78(1.28-2.48)$ & $1.41(0.91-2.18)$ & $41.4 \%$ \\
\hline \multicolumn{6}{|l|}{ Luminal B subtype } \\
\hline Normal weight & $44(41.1)$ & $44(34.4)$ & 1.00 & 1.00 & \\
\hline Overweight & $32(29.9)$ & $34(26.6)$ & $1.27(0.51-3.13)$ & $1.36(0.41-4.57)$ & \\
\hline Overweight & $9(30.0)$ & $10(20.8)$ & $0.60(0.10-3.45)$ & - & - \\
\hline Obese & $7(23.3)$ & $13(27.1)$ & $1.09(0.14-8.84)$ & & \\
\hline \multicolumn{6}{|c|}{ Triple-negative subtype } \\
\hline Normal weight & $33(39.8)$ & $44(38.9)$ & 1.00 & 1.00 & \\
\hline Overweight & $29(34.9)$ & $39(34.5)$ & $1.23(0.55-2.74)$ & $1.32(0.46-3.80)$ & - \\
\hline Obese & $21(25.3)$ & $30(26.6)$ & $1.18(0.54-2.57)$ & $1.04(0.42-2.54)$ & \\
\hline
\end{tabular}

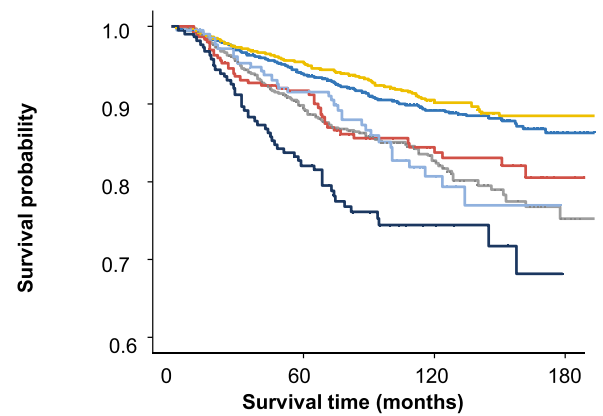

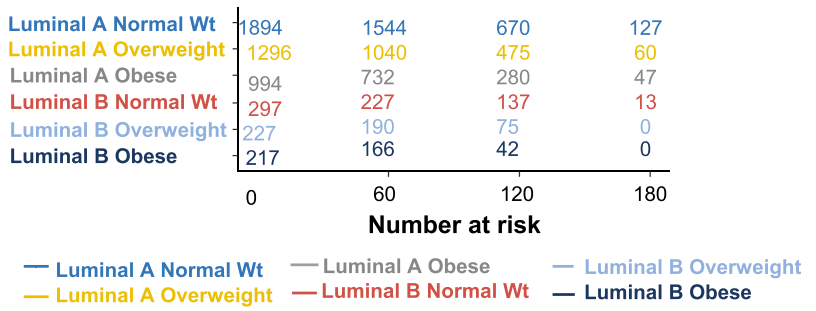

Fig. 2 Breast cancer-specific survival by body mass index categories for Luminal A-like and Luminal B-like tumor subtypes (adjusted for age at diagnosis)

Exposure, covariates, and outcome

Height and weight at clinical visits were abstracted from medical charts. Weight at diagnosis was available for $91.6 \%$ of women, prior to diagnosis only for $0.6 \%$ of women, and after diagnosis only for $7.8 \%$ of women. Missing weight at diagnosis was imputed for women with at least one other weight measurement (see "Statistical analyses" below). ${ }^{42}$ Weight and height were used to calculate BMI at diagnosis, which was categorized as normal weight $\left(18.5-24.9 \mathrm{~kg} / \mathrm{m}^{2}\right)$, overweight $\left(25-29.9 \mathrm{~kg} / \mathrm{m}^{2}\right)$, and obese $\left(\geq 30.0 \mathrm{~kg} / \mathrm{m}^{2}\right){ }^{43}$ Small cell sizes precluded examination of higher obesity levels (e.g., $\geq 35.0$ ) and underweight (BMl $\left.<18.5 \mathrm{~kg} / \mathrm{m}^{2}\right)$. Women completely missing height or at least a single weight in the medical records $(N=240$ cases; 188 subcohort) were excluded from analyses. Tumor size, node status, and metastases, tumor grade, treatment information, ${ }^{41}$ and Charlson comorbidities ${ }^{44}$ were abstracted from paper and electronic medical records. Tumor stage was categorized according to the American Joint Committee on Cancer V.6.

Biological markers determined by immunohistochemistry (IHC) including estrogen receptor (ER), progesterone receptor (PR), and Her2/neu (confirmed by fluorescent in situ hybridization) were abstracted from diagnostic pathology reports. IHC results were also obtained from tissue microarrays (TMAs) stained for ER, PR, and Her2/neu when missing. Women were considered ER or PR positive if $>1 \%$ IHC staining was present ${ }^{45}$ and Her2 positive if staining intensity was $>=3+$. Remaining values for women not included in TMAs (ER 6.7\%, PR 12.6\%, and Her2 24.7\%) were imputed. ${ }^{42}$ Breast cancer subtypes were categorized as: Luminal A like (ER or PR positive, Her2 negative), Luminal B like (ER or PR positive, Her2 positive), Her2 overexpressing (ER and PR negative, Her2 positive), and triple negative (ER, PR, and Her2 negative). ${ }^{18,46,47}$

The primary outcome of death from breast cancer was identified by the New Mexico Tumor Registry through linkages with the New Mexico Bureau of Vital Records and Health Statistics and the National Death Index. Underlying cause of death was coded using the International Classification of Diseases (ICD) Ninth and Tenth edition codes (ICD-9: 174 or ICD-10: C50.1-C50.9).

Multiple imputation methods

The sequence of regression models approach was used to impute missing values for all variables except body weight. ${ }^{42}$ A separate model was formulated for each variable, starting with an unconditional (mean only) model for the variable with the least missing and ending with a model for the variable with the most missing values, conditional on all other variables. (Fully imputed values are provided in Supplementary Table 1.) Variables used in the imputation included age at diagnosis, ethnicity, height, tumor size, lymph node status, tumor grade, ER, PR, Her2/neu, menopausal status, marital status, census tract income and education, 
receipt of chemotherapy, radiation and endocrine therapy, Charlson comorbidity conditions, ${ }^{44}$ vital status, and survival time.

For imputation of missing weight at diagnosis from other weight measurement(s), additional modeling took into account the timing of the additional measures. A subject's weight was allowed to vary over time according to an Ornstein-Uhlenbeck (O-U) process with subject-specific mean level as a function of available covariates. The $\mathrm{O}-\mathrm{U}$ process was evaluated on a month grid, using observed weight when available, thus making it equivalent to an autoregressive $(A R(1))$ model. ${ }^{48}$ Estimation and imputation for all variables were carried out simultaneously via a Bayesian computational framework (rjags package in R). To assess the performance of the weight imputation model, a cross-validation was run by predicting weight at diagnosis for $20 \%$ of women with a known weight. $R$-squared for the resulting prediction was 82.9 .

\section{Statistical analyses}

Statistical analyses were conducted using the standard case-cohort methodology. ${ }^{49}$ Women in the subcohort were weighted by the inverse of the sampling fraction $(100 \% / 15 \%=6.67)$. Multivariate Cox proportional hazards models were used to estimate HRs and $95 \%$ confidence intervals. The time scale was time in months from breast cancer diagnosis until date of last follow-up, death, or January 1, 2013. Breast cancer-specific death was the outcome of interest. All other causes of death or women alive at last follow-up were censored. Associations between BMI and breast cancer mortality were stratified by tumor molecular subtypes. Diagnosis age $(<40$, $40-49,50-59,60-69,70-79, \geq 80$ years) and Hispanic ethnicity were selected a priori to be included in regression models. There were no substantial changes to the estimates when age was included as a continuous variable in the model, thus results are based on the model including diagnosis age as a categorical variable. Linear trend was calculated by examining categorical variables as ordinal in the model.

To assess tumor characteristics as mediators of the BMI-survival relationship, we first examined whether higher BMI was associated with more aggressive tumor characteristics, which suggests that tumor features might be consequences of BMI. Specifically, we determined whether tumor stage and tumor grade, rather than being evaluated as potential confounders, might more appropriately be considered intermediate variables on the causal pathway (sometimes termed causal mediators) between BMI and mortality. As evidence, we assessed the temporal relationship (BMI exposure precedes each mediator), dose-response, and specificity of the relationship. We examined the cross-sectional association between BMI and potential mediators in polytomous logistic regression models, including only the population-based subcohort, adjusted for diagnosis age. Findings from these investigations were used to inform the analysis of the role of BMI in breast cancer survival.

Cox proportional hazards models for breast cancer-specific mortality were fit for the case-cohort to quantify the effect of BMI on mortality potentially mediated through tumor characteristics. We first calculated the age-adjusted association between BMI and mortality. Then we added tumor characteristics to the model. The mediation proportion, calculated using the difference in log-hazards (see p. 493 in ref. ${ }^{15}$ ), suggests the proportion of the BMI effect on mortality that acts through potential intermediates, such as tumor stage and grade.

We evaluated breast cancer-specific mortality within strata of tumor subtypes. We also evaluated whether the effect of BMI on breast cancerspecific mortality differed by subtype, by including the main effects (BMI and luminal vs. non-luminal subtype) and an interaction term (BMIX luminal subtype) in the Cox regression model.

The proportional hazards assumption was validated using Schoenfeld residuals. ${ }^{50}$ Confounding assumptions necessary for causal interpretation of the direct and indirect effect estimates were assessed. ${ }^{51}$ Analyses were conducted using SAS (version 9.4; Cary, N.C.) and R (v.3.4.3, Vienna, Austria). Final multiple imputation estimates were produced using SAS Proc Mlanalyze (20 imputations). Kaplan-Meier plots were produced in the $\mathrm{R}$ software. A two-sided test of statistical significance was defined as $p<$ 0.05. A Health Insurance Portability and Accountability Act waiver of consent was obtained for previously collected data. All study procedures were approved by the University of New Mexico Health Sciences Center institutional review board. Details regarding access to the data supporting this manuscript have been published. ${ }^{52}$

\section{Reporting summary}

Further information on research design is available in the Nature Research Reporting Summary linked to this article.

\section{DATA AVAILABILITY}

Aggregated patient data supporting all the figures, tables, and supplementary files in the published article are not publicly available to protect patient privacy but will be made available on request from the corresponding author, upon institutional review board approval as described at https://doi.org/10.6084/m9.figshare. $9428555 .{ }^{52}$ Release of vital status data is governed by the New Mexico state law, and therefore these data are not currently available. The data generated and analyzed during this study are described in the following data record: https://doi.org/10.6084/m9. figshare. $9428555 .^{52}$

\section{CODE AVAILABILITY}

Statistical code for imputation methods will be available in the pending papers.

Received: 23 April 2019; Accepted: 30 August 2019;

Published online: 02 October 2019

\section{REFERENCES}

1. Hales, C. M., Carroll, M. D., Fryar, C. D. \& Ogden, C. L. Prevalence of Obesity Among Adults and Youth: United States, 2015-2016. NCHS Data Brief 1-8 (2017).

2. Hales, C. M., Fryar, C. D., Carroll, M. D., Freedman, D. S. \& Ogden, C. L. Trends in obesity and severe obesity prevalence in US youth and adults by sex and age, 2007-2008 to 2015-2016. JAMA. https://doi.org/10.1001/jama.2018.3060 (2018).

3. Chan, D. S. et al. Body mass index and survival in women with breast cancersystematic literature review and meta-analysis of 82 follow-up studies. Ann. Oncol. 25, 1901-1914 (2014).

4. Protani, M., Coory, M. \& Martin, J. H. Effect of obesity on survival of women with breast cancer: systematic review and meta-analysis. Breast Cancer Res. Treat. 123, 627-635 (2010).

5. Warner, E. T. et al. Racial and ethnic differences in breast cancer survival: mediating effect of tumor characteristics and sociodemographic and treatment factors. J. Clin. Oncol. 33, 2254-2261 (2015).

6. Ellis, L. et al. Racial and ethnic disparities in cancer survival: the contribution of tumor, sociodemographic, institutional, and neighborhood characteristics. J. Clin. Oncol. https://doi.org/10.1200/JCO.2017.74.2049 (2017).

7. Sun, X. et al. Post-diagnosis adiposity and survival among breast cancer patients: influence of breast cancer subtype. Cancer Causes Control 26, 1803-1811 (2015).

8. Schisterman, E. F., Cole, S. R. \& Platt, R. W. Overadjustment bias and unnecessary adjustment in epidemiologic studies. Epidemiology 20, 488-495 (2009).

9. Cespedes Feliciano, E. M. et al. Body mass index, PAM50 subtype, recurrence, and survival among patients with nonmetastatic breast cancer. Cancer 123, 2535-2542 (2017).

10. Jeon, Y. W. et al. Relationship between body mass index and the expression of hormone receptors or human epidermal growth factor receptor 2 with respect to breast cancer survival. BMC Cancer 15, 865 (2015).

11. Jiralerspong, S. et al. Obesity, diabetes, and survival outcomes in a large cohort of early-stage breast cancer patients. Ann. Oncol. 24, 2506-2514 (2013).

12. Kawai, M. et al. Body mass index and survival after diagnosis of invasive breast cancer: a study based on the Japanese National Clinical Database-Breast Cancer Registry. Cancer Med. 5, 1328-1340 (2016).

13. Mowad, R. et al. Does obesity have an effect on outcomes in triple-negative breast cancer? J. Surg. Res. 184, 253-259 (2013).

14. Sparano, J. A. et al. Obesity at diagnosis is associated with inferior outcomes in hormone receptor-positive operable breast cancer. Cancer 118, 5937-5946 (2012).

15. VanderWeele, T. J. Explanation in Causal Inference: Methods for Mediation and Interaction. (Oxford University Press, 2015).

16. Ligibel, J. A. et al. Body mass index, PAM50 subtype, and outcomes in nodepositive breast cancer: CALGB 9741 (Alliance). J. Natl Cancer Inst. 107, https://doi. org/10.1093/jnci/djv179 (2015).

17. Sahin, $\mathrm{S}$. et al. The association between body mass index and immunohistochemical subtypes in breast cancer. Breast 32, 227-236 (2017).

18. Fontanella, C. et al. Impact of body mass index on neoadjuvant treatment outcome: a pooled analysis of eight prospective neoadjuvant breast cancer trials. Breast Cancer Res. Treat. 150, 127-139 (2015). 
19. Widschwendter, P. et al. The influence of obesity on survival in early, high-risk breast cancer: results from the randomized SUCCESS A trial. Breast Cancer Res. 17, 129 (2015).

20. Ewertz, M. et al. Effect of obesity on prognosis after early-stage breast cancer. J. Clin. Oncol. 29, 25-31 (2011).

21. Kamineni, A. et al. Body mass index, tumor characteristics, and prognosis following diagnosis of early-stage breast cancer in a mammographically screened population. Cancer Causes Control 24, 305-312 (2013).

22. Kwan, M. L. et al. Obesity and mortality after breast cancer by race/ethnicity: The California Breast Cancer Survivorship Consortium. Am. J. Epidemiol. 179, 95-111 (2014).

23. Neuhouser, M. L. et al. Overweight, obesity, and postmenopausal invasive breast cancer risk: a secondary analysis of the women's health initiative randomized clinical trials. JAMA Oncol. 1, 611-621 (2015).

24. Chlebowski, R. T. \& Reeves, M. M. Weight loss randomized intervention trials in female cancer survivors. J. Clin. Oncol. 34, 4238-4248 (2016).

25. Reeves, M. M., Terranova, C. O., Eakin, E. G. \& Demark-Wahnefried, W. Weight loss intervention trials in women with breast cancer: a systematic review. Obes. Rev. 15, 749-768 (2014).

26. Goodwin, P. J. \& Stambolic, V. Impact of the obesity epidemic on cancer. Annu. Rev. Med. 66, 281-296 (2015).

27. Hopkins, B. D., Goncalves, M. D. \& Cantley, L. C. Obesity and cancer mechanisms: cancer metabolism. J. Clin. Oncol. 34, 4277-4283 (2016).

28. lyengar, N. M., Gucalp, A., Dannenberg, A. J. \& Hudis, C. A. Obesity and cancer mechanisms: tumor microenvironment and inflammation. J. Clin. Oncol. 34, 4270-4276 (2016).

29. Kwan, M. L. et al. Association of high obesity with PAM50 breast cancer intrinsic subtypes and gene expression. BMC Cancer 15, 278 (2015).

30. Daling, J. R. et al. Relation of body mass index to tumor markers and survival among young women with invasive ductal breast carcinoma. Cancer 92, 720-729 (2001).

31. Cohen, S. S. et al. Obesity and screening for breast, cervical, and colorectal cancer in women: a review. Cancer 112, 1892-1904 (2008).

32. Fair, A. M. et al. Obesity, gynecological factors, and abnormal mammography follow-up in minority and medically underserved women. J. Womens Health (Larchmt.) 18, 1033-1039 (2009).

33. Majed, B. et al. Is obesity an independent prognosis factor in woman breast cancer? Breast Cancer Res. Treat. 111, 329-342 (2008).

34. lyengar, N. M., Hudis, C. A. \& Dannenberg, A. J. Obesity and cancer: local and systemic mechanisms. Annu. Rev. Med. 66, 297-309 (2015).

35. Brown, K. A. et al. Menopause is a determinant of breast aromatase expression and its associations with BMI, inflammation, and systemic markers. J. Clin. Endocrinol. Metab. 102, 1692-1701 (2017).

36. lyengar, N. M. et al. Menopause is a determinant of breast adipose inflammation. Cancer Prev. Res. (Phila.) 8, 349-358 (2015).

37. Bhardwaj, P. et al. Estrogens and breast cancer: mechanisms involved in obesityrelated development, growth and progression. J. Steroid Biochem. Mol. Biol. 189, 161-170 (2019)

38. Berger, N. A. Crown-like structures in breast adipose tissue from normal weight women: important impact. Cancer Prev. Res. (Phila.) 10, 223-225 (2017).

39. Bigornia, S. J. et al. Relation of depot-specific adipose inflammation to insulin resistance in human obesity. Nutr. Diabetes 2, e30 (2012).

40. Hill, D. A. et al. Estrogen receptor quantitative measures and breast cancer survival. Breast Cancer Res. Treat. 166, 855-864 (2017).

41. Hill, D. A. et al. Breast cancer survival, survival disparities, and guideline-based treatment. Breast Cancer Res. Treat. https://doi.org/10.1007/s10549-018-4761-7 (2018).

42. Raghunathan, T. E., Lepkowski, J. M., Van Hoewyk, J. \& Solenberger, P. A multivariate technique for multiply imputing missing values using a sequence of regression models. Surv. Methodol. 27, 85-96 (2001).

43. World Health Organization. Obesity and overweight fact sheet No. 311. http:// www.who.int/mediacentre/factsheets/fs311/en/index.html. Accessed May 2012.

44. Charlson, M. E., Pompei, P., Ales, K. L. \& MacKenzie, C. R. A new method of classifying prognostic comorbidity in longitudinal studies: development and validation. J. Chronic Dis. 40, 373-383 (1987).

45. Hammond, M. E. et al. American Society of Clinical Oncology/College Of American Pathologists guideline recommendations for immunohistochemical testing of estrogen and progesterone receptors in breast cancer. J. Clin. Oncol. 28, 2784-2795 (2010).

46. Chen, L. et al. Body mass index and risk of luminal, HER2-overexpressing, and triple negative breast cancer. Breast Cancer Res. Treat. 157, 545-554 (2016)

47. Howlader, N. et al. US incidence of breast cancer subtypes defined by joint hormone receptor and HER2 status. J. Natl Cancer Inst. 106, https://doi.org/ 10.1093/jnci/dju055 (2014).
48. Storlie, C. B., Branda, M. E., Gionfriddo, M. R., Shah, N. D. \& Rank, M. A. Prediction of individual outcomes for asthma sufferers. Biostatistics https://doi.org/10.1093/ biostatistics/kxx055 (2017).

49. Barlow, W. E. Robust variance estimation for the case-cohort design. Biometrics 50, 1064-1072 (1994)

50. Xue, X. et al. Testing the proportional hazards assumption in case-cohort analysis. BMC Med. Res. Methodol. 13, 88 (2013).

51. VanderWeele, T. J. Mediation analysis: a practitioner's guide. Annu Rev. Public Health 37, 17-32 (2016).

52. Blair, C. K. et al. Metadata supporting data files of the related article: Obesity and survival among a cohort of breast cancer patients: mediation by tumor characteristics. figshare. https://doi.org/10.6084/m9.figshare.9428555 (2019).

\section{ACKNOWLEDGEMENTS}

This work was supported by funding from the National Cancer Institute $(\mathrm{NCl})$ at the National Institutes of Health (R01CA132877 to D.A.H.) and the University of New Mexico (UNM) Comprehensive Cancer Center (NCI 2P30CA118100). The New Mexico Tumor Registry is funded by contract number HSN26120130010l-Task Order HHSN261000005 from the National Cancer Institute's Surveillance Epidemiology End Results (SEER) Program. In addition, the research in this paper was supported by the Human Tissue Repository and Tissue Analysis Shared Resource, funded by the UNM Department of Pathology and the UNM Comprehensive Cancer Center. This project was also supported in part by the Tobacco Settlement Funds from the University of New Mexico School of Medicine Research Allocation Committee. E.R.P. is supported by NIH R01 grants CA163890 and CA194496. C.K.B. is supported by an NIH K07 grant CA215937.

\section{AUTHOR CONTRIBUTIONS}

Study conception and design: C.K.B., C.L.W., A.M.N., E.R.P., L.C.L., M.R., D.A.H. Writinginitial draft: C.K.B., D.A.H. Data analysis: A.M.N., C.B.S., D.A.H. Data interpretation: A.M.N., C.B.S., D.A.H., C.K.B. Writing-final review and approval: C.K.B., C.L.W., A.M.N., C.B.S., E.R.P., L.C.L., M.R., D.A.H.

\section{COMPETING INTERESTS}

M.R. has provided consulting for TEVA Oncology, Celltrion. The other authors declare no competing interests.

\section{ADDITIONAL INFORMATION}

Supplementary information is available for this paper at https://doi.org/10.1038/ s41523-019-0128-4

Correspondence and requests for materials should be addressed to D.A.H.

Reprints and permission information is available at http://www.nature.com/ reprints

Publisher's note Springer Nature remains neutral with regard to jurisdictional claims in published maps and institutional affiliations.

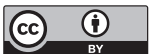

Open Access This article is licensed under a Creative Commons Attribution 4.0 International License, which permits use, sharing, adaptation, distribution and reproduction in any medium or format, as long as you give appropriate credit to the original author(s) and the source, provide a link to the Creative Commons license, and indicate if changes were made. The images or other third party material in this article are included in the article's Creative Commons license, unless indicated otherwise in a credit line to the material. If material is not included in the article's Creative Commons license and your intended use is not permitted by statutory regulation or exceeds the permitted use, you will need to obtain permission directly from the copyright holder. To view a copy of this license, visit http://creativecommons. org/licenses/by/4.0/.

(c) The Author(s) 2019 